\title{
ON ADAPTIVE CONTROL OF INFINITE DIMENSIONAL SYSTEMS
}

\author{
G. R. Sivaramakumar and K. Rajgopal
}

\author{
Department of Electrical Engineering \\ Indian Institute of Science, \\ Bangalore, 560 012, India.
}

Abstract

The problem of Self tuning control of a class of linear infinite dimensional systems is considered. A method is presented to design a stabilising finite dimenşional discrete time compensator for a known infinite dimensional system. To reduce the error in the parameter estimates due to ummodeled dynamics, an adaptive low pass filter is proposed to be used prior to the parameter estimator and conditions are presented for stable operation. The global stability is also discussed.

\section{Introduction}

Many physical systems in practice are governed by partial differential equations and hence infinite dimensional. The main problem in designing physically realizable stabilizing controller is 'spill-over' i.e., the interaction between the residual unmodeled dynamics and the controller giving rise to instability[2]. The main objective of this paper is to analyse this issue in the context of adaptive control and develop a method to reduce the effect of the spill- over.

For a known plant, three constructive schemes have been proposed by researchers [9],[6],[7] to design finite dimensional compensator with out spill-over. This paper deals with the adaptive controller of infinite dimensional systems based on the method of Curtain [6]. Essentially, our approach consists of estimation of the parameters of the original partial differential equation that governs the process and design of the controller based on Curtain's method. To reduce the error due to unmodeled dynamics, an adaptive low pass filter is proposed to be used prior to the parameter estimator and conditions are derived for the stable operation. The global stability is also discussed along the lines of Bai and Sastry [1]. For detailed proofs please refer [10].

\section{Discrete time finite dimensional compensators for infinite} dimensional systems.

All the methods used for the construction of finite order compensator design for infinite dimensional systems are based on reduced order approximation (ROM) of the plant. These methods suffer from the drawback of spill-over. To circumvent this problem Curtain has proposed a method along with some formulas to monitor the closed loop stability during the design process. Since adaptive control is generally implemented in discrete time, we extend Curtain's method to discrete time i.d.s in this section.

The advantages of Curtain's method are

(i) Computationally it is inexpensive.

(ii) Order of the compensator will be nominal and close to the number of unstable eigenvalues.

(iii) Closed loop stability can be assessed easily using explicit formulas.

The results, we derive, ane applicable to a class of i.d.s called 'Spectral Systems'. For the details, the reader is referred to [5].

3. Stability of discrete time systems

Consider a class of spectral systems described by

$$
\dot{x}=A x+B u ; y=C x
$$

where $x \in D(A)$ dense in Hilbert space $H$ endowed with a suitable norm and inner product. Let $T(t)$ be the strongly continuous semigroup generated by $A$ in $H$ such that $\|T(t)\| \leq M e^{\omega *}, M>0, \omega<\infty$.

Consider the Cauchy problem $\dot{x}=A x$. The solution of for this problem (in the mild sense) is given by

$$
x(t)=T(t) x_{0} \text { where } t \in[0, \infty)
$$

Proposition 1.1 [8]

Let $\tau>0$ and $X=\left\{x_{k}\right\}_{k=0}^{\infty}$ be a sequence of elements in $H$. Assume $A=T(\tau)$. Then the sequence $X$ satisfies the linear difference equation $x_{k+1}=A x_{k}$ for all $k \in Z_{0}^{+}$if and only if $x_{k}=x(k \tau)$ for all $k \in Z_{0}^{+}$where $x(t)=T(t) x_{0}$

CH3229-2/92/0000-3519\$1.00 @ 1992 IEEE
Instead of using $\hat{A}$ we use $A$ here onwands. A fundamental theorem on the growth propenty of the system $x_{k+1}=A x_{k}$ where $A$ is the linear bounded operator (since $T(t)$ is bounded for bounded $t$ ) and $A=T(\tau)$ is given in [8] as follows.

Theorem 3.1: Let $x_{k+1}=A x_{k}$ where $A$ is the linear bounded operator given by sampling of a system $\dot{x}=S x, x \in H$ and $T(t)$ is the semigroup generated by $S$, and $A=T(\tau), \tau>0$ and $x_{k}=x(k \tau), k \in N$ then $\exists M>1, r>0$ such that $\left\|A^{k}\right\| \leq M r^{k}$

Consider a bounded perturbation $\triangle A$ in $A$. Then the perturbed discrete time system is described as

$$
x_{k+1}=(A+\Delta A) x_{k}
$$

We have the following result.

Theorem 3.2 Let $x_{k+1}=(A+\Delta A) x_{k}$. Let the growth rate of a perturbation operator $\Delta A$ be governed by $\left\|(\Delta A)^{k}\right\| \leq \hat{M}^{k}$ where $M>1$ and $\hat{r}>0$. Then the growth rate of the perturbed system is govemed by $\left\|(A+\Delta A)^{k}\right\| \leq M M(r+\hat{r})^{k}$.

The proof is based on expanding $(A+\Delta A)^{k}$ as binomial series and application of Schwartz and Holder inequalities.

Now, we have a useful corollary.

Corollary 3.1: Let $x_{k+1}=A x_{k}$ be a stable system such that $\left\|A^{k}\right\| \leq M r^{k}, M>1, r<1$, and $\Delta A$ be such that $\left\|(\Delta A)^{k}\right\| \leq M r r^{k}$ If $\hat{r} \leq 1-r$ implying $\|\Delta A\|$ sufficiently small, then $x_{k+1}=(A+\Delta A) x_{k}$ is stable.

Based on the above corollary, Curtain's method can be extended to discrete time i.d.s. We just outline the procedure for the sake of completion.

Let $x_{k+1}=A x_{k}+B u_{k} ; y=C x_{k}$ be a discrete time version of a spectral system. A, B, C are bounded linear operators. The construction of the compensator is based on the modal decomposition of the state space $H=H^{p}+H^{s}$ where $H^{p}$ belong to unstable eigenvalues and $H^{s}$ belong to stable eigenvalues. Spectral systems allow such a decomposition Then A, B, C can be decomposed as

$$
A=\left[\begin{array}{rr}
A_{p} & 0 \\
0 & A_{s}
\end{array}\right] ; B=\left[\begin{array}{l}
B_{p} \\
B_{s}
\end{array}\right] ; C=\left[\begin{array}{ll}
C_{p} & C_{s}
\end{array}\right] .
$$

Assuming $A_{p}, B_{p}, C_{p}$ minimal, we can choose $F_{p}$ and $G_{p}$ such that $A_{p}+B_{p} F_{p}$ and $A_{p}+G_{p} C_{p}$ are stable with sufficient stability margin. Let $\pi_{p}$ be the projection operator on $H^{p}$ and $\pi_{n}$ on $H^{n}$-a modal subspace of $H$ and $n \geq p$.

Let $R_{n}$ be the isomorphism between $H^{n}$ and $R^{n}$. Then the nth order compensator is given by $F(z I-M)^{-1} G$ where

$$
\begin{aligned}
& M=R_{n}\left(A+\pi_{n} B F_{o}+G_{o} C\right) R_{n}^{-1} \\
& F=F_{o} R_{n}^{-1} ; F_{o}=\left(F_{p}, 0\right) ; G=-R_{n} G o ; G_{o}=\left[\begin{array}{c}
G_{p} \\
0
\end{array}\right]
\end{aligned}
$$

The compensator $(F, M, G)$ stabilizes the plant for large but finite $n$. The proof is based on theorem 3.2 and corollary 3.1. and arguments given for continuous time systems in [6]. To check the resulting closed loop stability, the formulas given in [6] can be used with obvious modifications to discrete time systems.

By proper choice of $p, F_{0}$ and $G_{o}$ it is possible to design the compensator with desired stability margin. The global stability can be argued if the estimated parameters converge to the neighbourhood of the actual parameters. Hence parameter estimation problem of i.d.s is examined in the next section. 


\section{Parameter Estimation}

In this section we analyse the problem of estimation of the coefficients in the p.d.e that governs the process from input - output data. The identification problem can be posed as follows.

Consider

$$
\dot{x}=A(q) x+B(q) \text { u } y=C(q) x ; x(0)=x_{0} \in H
$$

$q$ is the finite parameter set. For each $q \in Q \subset R^{k_{1}} A(q)$ is the infinitesimal generator of a strongly continuous semigroup $\{T(t: q)\}$ $\epsilon \geq 0$ on a Hilbert space $\boldsymbol{H}$.

The problem is to identify ' $q$ ' in equation (4.1) from the observa tions. The measurements $\hat{y}$ are available for all $t_{i}, 0 \leq t_{i} \leq T$ at different time instants. The value of ' $q$ ' is to be estimated by minimising

$$
J=\sum_{i-r}^{t}\left(\hat{v}\left(t_{i}\right)-\left(y\left(t_{i}\right)\right)^{2}\right.
$$

over $q \in Q$ subject to (4.1) using gradient algorithms. To compute $y\left(t_{i}\right)$ it is necessary to use some approximation technique and here we analyse 'Modal approximation' scheme.

\section{Let the ROM be}

$$
\dot{x}^{N}=A^{N}(q) x^{N}+B^{N}(q) u ; y^{N}=C^{N}(q) x^{N} ; x^{N}(0)=P^{N}(q) x_{0}(q)(4.3)
$$

where $P^{N}(q)$ is the projection operator. The approximate identification problem is as follows. Given $\hat{y}=\left\{\hat{y}\left(t_{i}\right)\right\}$ minimize $J^{N}(q)=J\left(q, x^{N}(, q), y^{N}\left(t_{i}\right)\right)$ over $q \in Q$ subject to $x^{N}$ satisfying (4.3). Let the solution be $q^{N}$.

Banks [4] has given a convergence theorem for the above approximation scheme for the convergence of $q^{N}$ to $q$ i.e., as $N \rightarrow \infty, q^{N} \longrightarrow q$.

We have the following observations.

Observation 1.

For the given $N$, the error in the parameter estimates $\left(q-q^{N)}\right.$ is due to approximation of $(C, A, B)$ by its ROM $\left(C^{N \cdot} A^{N} \cdot B^{N)}\right.$. Let $(\Delta C, \Delta A, \Delta B)$ be the residual subsystem which has been discarded in the estimation process.

Observation 2

In the case of spectral systems, the spectrum of $\Delta A$ are located fairly away from imaginary axis with natural frequencies in ascending order. These modes respond only to high frequency components of the input signal.

Instead of the measured output, if the output from the ROM $\left(y^{r}\right)$ of the plant had been used for parameter estimation, then the exact values of ' $q$ ' could be arrived at. Hence, if we have a low pass filter which has cutoff frequency corresponding to Nth order mode of the system, the error $q-q^{N}$ will be drastically reduced.

However, design of 1.p.f requires the knowledge of unknown Nth order mode. Hence, an approximate l.p.f can be inserted prior to the parameter estimator and then the l.p.f parameters can be updated with new estimates. The scheme is shown in figure 1 . It can be seen that a closed loop system is getting formed among the parameter estimator 1.p.adaptive filter, and the plant. For proper operation, this loop should be stable. Here, we outline the steps involved in deriving the conditions for the stable operation.

The steps are as follows.

(1) The error in the parameter estimates of the ROM due to residual components is evaluated.

(2) Due to the error in the estimated parameters, there will be an error in the eigenvalues and hence the largest eigen-frequency. An upper bound for the difference between the estimated cutoff frequency of the filter and the exact frequency corresponding to Nth order mode is obtained.

(3) From the expression for the evolution of the squared norm of the output error, it is seen that for the boundedness of all the signals in the identifier loop, there should be sufficient excitation at the modeled frequency range, low excitation in the error range, high onder of approximation and good initial estimates.

Once the parameters are estimated to a sufficient accuracy, then the compensator can be constructed as in section 3 . Then it can be shown that the overall system is stable if parameters converge to sufficiently small neighbourhor's of the exact parameters.

\section{S. Stability}

Here we just outline how we go about analysing overall global stability. It can be shown that the plant along with the controller is described by

$$
\bar{x}_{k+1}=\left(\bar{A}+\Delta \bar{A}_{k}\right) \bar{x}_{k}
$$

where $\bar{x}$ is the vector of the plant states and the controller states. $\bar{A}$ will be stable if the estimated parameters converge sufficiently close to the neighbourhood of the exact values and the controller is designed with sufficient stability margin. $(\Delta A)_{k}$ is the error in the estimation process which goes to zero as $k$ goes to $\infty$. It can be shown the above system is exponentially stable by extending the global stability theorem of $\mathrm{Bai}$ and Sastry[1] to discrete time i.d.s.

\section{Conclusion}

A Method is presented for adaptive control of linear i.d.s. incorporating a new adaptive filter in the parameter estimator loop and conditions are derived for the stable operation. The global stability is also discussed. Application of these ideas to the control of large space structures deserves detailed investigation which is under progness.

\section{References}

1) E.W.Bai and S.Sastry, "Global Stability Proofs for Continuous Time Indirect Adaptive Control Systems", IEEE Trans. on Automatic Control., Vol AC-32, No 6, 1987.

2) M.J.Balas, "Trends in Large Space Structures Control Theory, Fondest Hopes and Wildest Dreams", IEEE Trans. on Automatic Control Vol AC-27, No. 3, 1982.

3) M.J.Balas and C.R.Johnson, "Adaptive Control of Distributed Parameter Systems: The Ultimate Reduced Order Problem", Proc. IEEE CDC, 1979.

4) H.T.Banks and K.Kunisch, "An Approximation Theory for Nonlinear Partial Differential Equations with Application to Identification and Controls", SIAM J. of Control and Optimisaiun, Vol 20, 6, 1982.

5) R.F.Curtain, "Spectral Systems", International Journal of Control. vol-39, pp.657-666, 1984

6) R.F.Curtain, "Pole Assignment for Distributed systems by Finite Dimensional Control", Automatica, vol 21, no 1, pp.57-67, 1985.

7) J.S.Gibson, and M.Navid, "Optimal Control of Flexible Structures", in Proc. of 20th IEEE CDC, pp.700-701, 1981.

8) K.Machejprezylinsky, "Stability of Linear Infinite Dimensional Systems - Revisited", International Journal of Control, vol 48, no 2, 1988.

9) J.M.Schumaker, "A Direct Approach to Compensator Design for Distributed Parameter Systems", SIAM J. of Control and Optimisation, vol 21, 823

10) G. R. Sivaramakumar, "Model Matching and Adaptive Control of Finite and Infinite Dimensional Systems", PhD. Thesis, Dept. of Elect. Engg., Indian Institute of Science, (submitted).

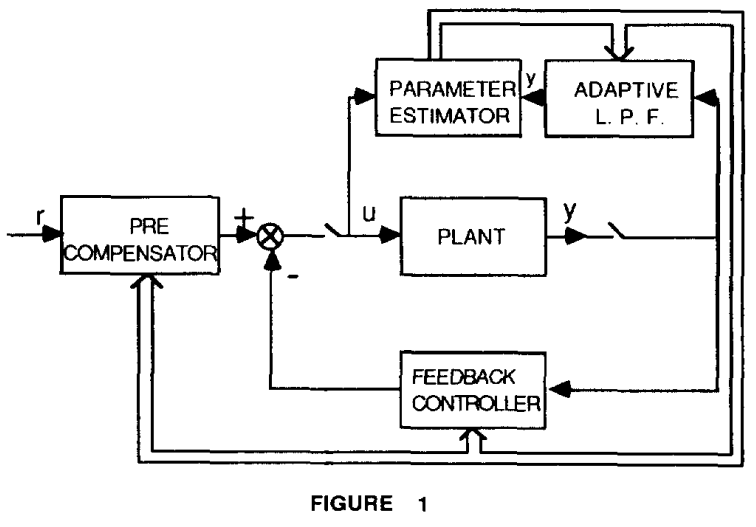

ADAPTIVE CONTROL WITH ADAPTIVE L.P.F 